\title{
Is Bitcoin a hedge or safe haven for currencies? An intraday analysis
}

Article

Accepted Version

Creative Commons: Attribution-Noncommercial-No Derivative Works 4.0

Urquhart, A. and Zhang, H. (2019) Is Bitcoin a hedge or safe haven for currencies? An intraday analysis. International Review of Financial Analysis, 63. pp. 49-57. ISSN 1057-5219 doi: https://doi.org/10.1016/j.irfa.2019.02.009 Available at https://centaur.reading.ac.uk/82426/

It is advisable to refer to the publisher's version if you intend to cite from the work. See Guidance on citing.

To link to this article DOI: http://dx.doi.org/10.1016/j.irfa.2019.02.009

Publisher: Elsevier

All outputs in CentAUR are protected by Intellectual Property Rights law, including copyright law. Copyright and IPR is retained by the creators or other copyright holders. Terms and conditions for use of this material are defined in the End User Agreement.

\section{www.reading.ac.uk/centaur}

\section{CentAUR}

Central Archive at the University of Reading

Reading's research outputs online 


\title{
Is Bitcoin a hedge or safe haven for currencies? An intraday analysis
}

\begin{abstract}
Bitcoin has attracted a wealth of attention in the media and by investors alike and this paper investigates whether Bitcoin can act as a hedge or safe haven against world currencies. Contrary to previous studies, we assess the relationship between Bitcoin and currencies at the hourly frequency since Bitcoin experiences quite large volatility throughout the day. We employ an ADCC model and find that Bitcoin can be an intraday hedge for the CHF, EUR and GBP, but acts as a diversifier for the AUD, CAD and JPY. We also implement the non-temporal Hansen (2000) test to examine the safe haven properties of Bitcoin and find that Bitcoin is a safe haven during periods of extreme market turmoil for the CAD, CHF and GBP. Therefore our results indicate that Bitcoin does act as an intraday hedge, diversifier and safe haven for certain currencies, which will be of great interest to currency, cryptocurrency and high-frequency investors alike.
\end{abstract}

Keywords: safe haven; hedge; Bitcoin; high-frequency; dynamic conditional correlation

JEL Classification: G10, G11, G14, G15 


\section{Introduction}

Bitcoin, first proposed by Nakamoto (2008), is a new financial asset which is a peer-to-peer electronic cash system which allows online payments to be sent directly from one party to another without going through a financial institution. Therefore unlike most other financial assets, Bitcoin has no association with any authority and has no physical representation. Bitcoin's value is not based on any tangible asset or any country's economy and instead is based upon the security of an algorithm which traces all transactions. The potential use of Bitcoin as a medium of exchange is attractive due to its low transaction costs, its peer-to-peer, global and government-free design. However users may be concerned by the lack of confidence in the system as well as the lack of acceptability of Bitcoin to make transactions. ${ }^{1}$ Another concern for users of Bitcoin is the volatility of Bitcoin which is represented by the Bitcoin price increase from $\$ 4.9$ in September 2011 to over $\$ 6,000$ by October 2017. Nevertheless, Bitcoin has received a lot of attention by investors, media and politicians.

The dramatic growth of Bitcoin (and other cryptocurrencies) challenge politicians and policymakers as Bitcoin resembles the role of money but also creates an alternative environment for businesses. Bitcoin has recently experienced excessive speculative demand which supported empirically by Baur et al (2018) who report that Bitcoin accounts are mainly used as a speculative investment rather than an alternative currency or medium of exchange. Given the surge in price, Bitcoin has become an increasingly important and popular topic which has been widely covered by the media, government bodies and the academic community. However Bitcoin has not been examined in much detail in academia given the media attention, and it is an area that needs to be addressed in future research (Lucey et al 2018).

A group of recent papers examine the price dynamics and speculation bubbles in cryptocurrency markets, although the results are somewhat mixed. ${ }^{2}$ Cheah and Fry (2015) show that cryptocurrencies are prone to substantial speculative bubbles and that the fundamental value of Bitcoin is zero, while recently Corbet et al (2018a) examine the fundamental drivers of the Bitcoin price and show there are clear periods of bubble behaviour of Bitcoin. Dwyer (2015) shows that the average monthly volatility of Bitcoin is higher than that of gold and any currency, while the lowest monthly volatility of Bitcoin is less than the highest monthly volatility of gold and any currency. Urquhart (2017) shows price clustering in Bitcoin while Katsiampa (2017) shows the

\footnotetext{
${ }^{1}$ However some companies do accept Bitcoin as a form of exchange, such as Microsoft, Dell, Expedia, Tesla and many more.

${ }^{2}$ For a complete review of the literature of cryptocurrencies, see Corbet et al (2018b).
} 
importance of the short- and long-run component of the conditional variance when examining the volatility of Bitcoin through various GARCH models. Peng et al (2018) examine the volatility of three cryptocurrencies and show that SVR-GARCH models outperformance traditional GARCH models for daily and hourly data and therefore machine learning models yield better forecasting power for all three cryptocurrencies examined. The market efficiency of Bitcoin has also been examined in some detail, with Urquhart (2016) first documenting the inefficiency of Bitcoin returns which has subsequently been supported by Nadarajah and Chu (2017), Bariviera (2017) and Tiwari et al (2017). Recently, Urquhart (2018) shows that high trading volume and volatility are drivers of the attention of Bitcoin while Shen et al (2019) show that the number of tweets related to Bitcoin significant predict the trading volume and volatility of Bitcoin

An area of research which has received some attention in the literature is the relationship between Bitcoin and other financial assets and determining whether Bitcoin can be classified as a diversifier, hedge or safe haven against other financial assets. Dyhrberg (2016) shows that Bitcoin can act as a hedge against the US dollar and the UK stock market, sharing similar hedging capabilities to gold. Bouri et al (2017a) employ a quantile regression approach to analyse the relationship between gold and global uncertainty and show that Bitcoin can hedge against global uncertainty at short investment horizons and in bull regimes only. Also, Bouri et al (2017b) employ a DCC model and show limited evidence of the hedging and safe properties of Bitcoin, although it can still be an effective diversifier. Corbet et al (2018c) suggest that Bitcoin has a role in an investor's portfolio, although Bitcoin may contain its own idiosyncratic risks that are difficult to hedge against, while Shahzad et al (2019) show that Bitcoin can be a safe haven, although its role is time-varying and differ across markets. Recently, Plantakis and Urquhart (2019) show that add cryptocurrencies to stock-bond-commodity portfolios significantly improves the portfolio performance by much higher risk-adjusted returns, which is confirmed by Kajtazi and Moro (2019). Therefore there is some evidence that Bitcoin has some hedging capabilities and diversification benefits. All of the aforementioned papers have examined the interaction between Bitcoin and other financial assets at the daily frequency. However given the rise in high-frequency trading amongst investors and the huge variation in price of Bitcoin at the intraday level, the interaction between Bitcoin and other financial assets at the daily level may be quite different to the intraday interaction between the assets.

The hedging and safe haven properties of gold have been examined in some detail with Baur and Lucey (2010) find that gold is a hedge and safe haven for stocks but not for bonds, while gold is 
also found to act as a safe haven for only 15 days after a market crash. Baur and McDermott (2010) extend this analysis and show gold's status as a safe haven for equities but not for all countries examined. Bredin et al (2015) employ a wavelet analysis and show that gold can be safe haven up to a year while Lucey and $\mathrm{Li}$ (2014) find the instability of the safe haven property of gold, indicating that gold's ability to act as a hedge and safe haven fluctuate over time. Ciner et al (2013) report that gold can act as a safe haven for the US dollar and UK pound from 2000 onwards. Therefore is a well-established literature examining the hedge and safe haven properties of gold but there lacks a detailed examination of Bitcoin. ${ }^{3}$

This paper adds to the cryptocurrency and safe haven literature by examining the hedge and safe haven properties of Bitcoin and currencies at the intraday level. Especially, we study the hourly frequency of Bitcoin and six developed currencies to determine whether Bitcoin can be classified as a diversifier, hedge or safe haven asset for popular currencies. Given the increased in Bitcoin trading as well as the evidence that there is value in including Bitcoin in a well-diversified portfolio, we study whether investors are jumping to Bitcoin when there are extreme returns in developed currencies. We choose currencies since unlike equities, since they trade 24-hours a day and therefore are more aligned to the trading hours of Bitcoin. ${ }^{4}$ We employ a DCC model to determine the time-varying hedge properties of Bitcoin while we also follow Fatum and Yamamoto (2016) by employing the non-temporal threshold testing procedure originally developed by Hansen (2000) which enables us to endogenously identify the market thresholds of extreme price movements. Specifically, we examine periods of extreme returns in each currency and study the behaviour of Bitcoin returns during this period. This enables us to examine the safe haven behaviour of investors when there are extreme negative returns in each developed currency. Our results suggest that Bitcoin is a hedge at various times for each currency according to the asymmetric dynamic conditional correlation (ADCC) model at the hourly frequency indicating that investors should include Bitcoin in their portfolios to help diversify against currency risk. Using regression analysis, we show that over the full sample period Bitcoin is a hedge for the CHF, EUR and GBP, while a diversifier for the AUD, JPY and CAD currencies. Bitcoin is also a safe haven for the CAD, CHF and GBP currencies indicating that investors may want to take the 'flight to safety' during periods of extreme market turmoil in each of these currencies. Our results will therefore be of great interest to investors of Bitcoin who include the cryptocurrency in their portfolios. Recently, Platanakis and Urquhart (2019) show that the inclusion of Bitcoin in a simple stock bond portfolio

\footnotetext{
${ }^{3}$ For a complete review of the literature of gold, including the hedge and safe haven properties, see O'Connor et al (2015).

${ }^{4}$ Bitcoin markets trade 24-hours a day, 7 days a week.
} 
significantly improves the risk-return metrics, even when Bitcoin experiences negative returns. Platanakis et al (2018) examine the diversification benefits of cryptocurrencies and show that there is little difference between optimal and naïve diversification. Therefore our paper adds to these papers by examining the intraday hedge and safe haven behaviour of Bitcoin to currencies where our results could be incorporated by investors who employ an intraday algorithm to trade their portfolios.

This paper contributes to the growing literature on Bitcoin in the following ways. This is the first paper to thoroughly examine the hedge and safe haven properties of Bitcoin at the intraday level. Previous research has reported the potential hedging properties of Bitcoin at the daily level but no paper has examined the intraday hedging for safe haven properties of Bitcoin. Given the rise in high-frequency trading and the high volatility in intraday Bitcoin returns, examining hourly data will provide a more comprehensive indication of the relationship between Bitcoin and world currencies. We also employ a recently developed methodology to examine the safe haven properties of Bitcoin, which is able to endogenously identify periods high market turmoil. This ensures we only examine periods of extreme currency returns in order to determine how Bitcoin reacts in periods of currency market turmoil.

The rest of the paper is organized as follows. Section 2 discusses the data employed in this paper while Section 3 presents the methodology. Section 4 reports the empirical results while Section 5 provides conclusions.

\section{Data}

We collect high-frequency Bitcoin data from www.bitcoincharts.com which provides data on a number of liquid Bitcoin markets. We select the Bitcoin price from the Bitstamp exchange since it is one of the first, most popular and liquid Bitcoin exchanges (Brandvold et al (2015)). Bitstamp is also one of the largest Bitcoin exchanges, is based in the UK and is considered to be a rather safe exchange by market participants around the world (Bouri et al (2017a)). We study intraday Bitcoin data from $1^{\text {st }}$ November 2014 to $31^{\text {st }}$ October 2017 thereby incorporating three full years of intraday data. The start date is selected since before this date, intraday Bitcoin data was quite illiquid with insubstantial trading volume while the end date ensures we avoid any seasonality effects. We select the hourly frequency since any higher frequency for Bitcoin lacks liquidity. Hourly currency data is provided from Bloomberg for the six main developed currencies throughout the world, namely the Australian dollar (AUD), Canadian dollar (CAD), the Swiss 
franc (CHF), the Euro (EUR), the British pound (GBP) and the Japanese yen (JPY), all per one US dollars (USD). Therefore if the value of the non-US currency increases, this suggests a weakening of the non-US currency and vice-versa. Consistent with Manahov et al (2014) we allow 24-hour trading for currencies except at weekends with trading taking place from $9 \mathrm{pm}$ (GMT) on Sunday until $10 \mathrm{pm}$ on Friday. Since Bitcoin trades 24-hour a day, 7-days a week, we filter out Bitcoin prices during periods when the currency markets are closed. ${ }^{5}$ Figure 1 shows the timeseries plot of the currencies, per US dollars, over time while Figure 2 reports the time series graph of the Bitcoin price over the same sample period. We employ logarithmic returns for Bitcoin and currencies such that:

$$
r_{t}=\left[\ln \frac{P_{t}}{P_{t-1}}\right] \times 100
$$

Where $r_{t}$ are the logarithmic returns and $P_{t}$ is the price at time $t$ and $P_{t-1}$ is the price at time $t-1$. The descriptive statistics of the returns are reported in Table 1 where we can see that Bitcoin has largest return, which is over 15 times larger than any of the currencies. All currencies experience positive returns, which indicates a weakening of the currency against the US dollar. The currency with the highest mean return is the GBP while JPY has the smallest mean return. Bitcoin is substantially the most volatile of all currencies while CHF is the most volatile currency while CAD is the least volatile currency. All variables experience negative skewness except the GBP and all currencies experience excess kurtosis indicating a leptokurtic distribution. Table 2 reports the correlation matrix between the variables and it shows that Bitcoin has very small correlations with all currencies. The correlation between AUD and Bitcoin and JPY and Bitcoin is - 0.01 indicating a negative relationship between these two currencies and Bitcoin. However the correlation between the remaining the currencies and Bitcoin is all 0.00. Although the correlation matrix gives some information into the relationship between these variables, our detailed analysis below provides a more robust estimation of the relationship between Bitcoin and currencies.

\section{Methodology}

We follow the definitions of Baur and Lucey (2010) to define a hedge, diversifier and safe haven since these have become standard in the literature. A hedge is defined as an asset that is uncorrelated or negatively correlated with another asset, while a diversifier is defined as an asset

\footnotetext{
${ }^{5}$ The trading volume of Bitcoin and the currencies is extremely small during the periods excluded (Friday $10 \mathrm{pm}$ to Sunday $9 \mathrm{pm}$ ) and therefore all markets are illiquidity during this period.
} 
that is positively (but not perfectly) correlated with another asset. A safe haven asset is an asset that is uncorrelated or negatively correlated with another asset in times of market stress or turmoil. In this paper, the dynamic conditional correlation (DCC) model of Engle (2002) and the asymmetric dynamic conditional correlation (ADCC) model of Cappiello et al (2006) are used to model the volatility dynamics, conditional correlations and hedge ratios between Bitcoin and foreign exchange markets.

Let $r_{t}$ be a $n \times 1$ vector of asset returns. An $\mathrm{AR}(1)$ process for $r_{t}$ conditional on the information set $I_{t-1}$ can be written as:

$$
r_{t}=\mu+a r_{t-1}+\varepsilon_{t}
$$

The residuals are modelled as:

$$
\varepsilon_{t}=H_{t}^{1 / 2} z_{t}
$$

$H_{t}$ is the conditional covariance matrix of $r_{t}$ and $z_{t}$ is a $n \times 1$ i.i.d random vector of errors. The Engle (2002) dynamic conditional correlation (DCC) model is estimated in two steps, where in the first step, the GARCH parameters are estimated and the conditional correlations are estimated in the second step where:

$$
H_{t}=D_{t} R_{t} D_{t}
$$

Where $H_{t}$ is a $n \times n$ conditional covariance matrix, $R_{t}$ is the conditional correlation matrix, and $D_{t}$ is the diagonal matrix with time-varying standard deviations on the diagonal.

$$
D_{t}=\operatorname{diag}\left(h_{1, t}^{1 / 2}, \ldots h_{n, t}^{1 / 2}\right)
$$

$$
R_{t}=\operatorname{diag}\left(q_{1, t}^{-1 / 2}, \ldots q_{n, t}^{-1 / 2}\right) Q_{t} \operatorname{diag}\left(q_{1, t}^{-1 / 2}, \ldots q_{n, t}^{-1 / 2}\right)
$$


The expressions for $h$ are univariate GARCH ( $\mathrm{H}$ is a diagonal matrix). For the GARCH(1,1) model the elements of $H_{t}$ can be written as:

$$
h_{i, t}=\omega_{i}+\alpha_{i} \varepsilon_{i, t-1}^{2}+\beta_{i} h_{i, t-1}
$$

And that $Q_{t}$ is a symmetric positive definite matrix such that:

$$
Q_{t}=\left(1-\theta_{1}-\theta_{2}\right) \bar{Q}+\theta_{1} z_{t} z_{t-1}^{\prime}+\theta_{2} Q_{t-1}
$$

Where $\bar{Q}$ is the $n \times n$ unconditional correlation matrix of the standardized residuals $z_{i, t}\left(z_{i, t}=\frac{\varepsilon_{i, t}}{\sqrt{h_{i, t}}}\right)$. The parameters $\theta_{1}$ and $\theta_{2}$ are non-negative and these parameters are associated with the exponential smoothing process that is used to construct the dynamic conditional correlations. The DCC model is mean reverting as long as $\theta_{1}+\theta_{2}<1$. The correlation estimator is:

$$
\rho_{i, j, t}=\frac{q_{i, j, t}}{\sqrt{q_{i, i, t} q_{j, j, t}}}
$$

Cappiello et al (2006) build on the DCC model and the asymmetric GARCH model of Glosten et al (1993) by adding in an asymmetric term and create the Asymmetric DCC (ADCC) model such that:

$$
h_{i, t}=\omega_{i}+\alpha_{i} \varepsilon_{i, t-1}^{2}+\beta_{i} h_{i, t-1}+d_{i} \varepsilon_{i, t-1}^{2} I\left(\varepsilon_{i, t-1}\right)
$$

The indicator function $I\left(\varepsilon_{i, t-1}\right)$ is equal to one if $\varepsilon_{i, t-1}<0$ and 0 otherwise. For this specification, a positive value for $d$ means that the negative residuals tend to increase the variance more than positive ones. The asymmetric effect (or leverage effect) is designed to capture an often observed characteristic of financial assets that an expected fall in prices tends to increase volatility more than an unexpected increase in asset prices of the same magnitude. This can be interpreted 
that bad news increases volatility more than good news. For the ADDC model, the dynamics of $Q$ are given by:

$$
Q_{t}=\left(\bar{Q}-A^{\prime} \bar{Q} A-B^{\prime} \bar{Q} B-G^{\prime} \bar{Q}^{-} G\right)+A^{\prime} z_{t-1} z_{t-1}^{\prime} A+B^{\prime} Q_{t-1} B+G^{\prime} z_{t}^{-} z_{t}^{\prime-} G
$$

Where $A, B$ and $G$ are $n \times n$ parameters matrices and $z_{t}^{-}$are zero-threshold standardized errors which are equal to $z_{t}$ when less than zero and zero otherwise. $\bar{Q}$ and $\bar{Q}^{-}$are the unconditional matrices of $z_{t}$ and $z_{t}^{-}$respectively.

The methodology we employ to examine the safe haven properties of Bitcoin broadly follows the work of Fatum and Yamamoto (2016). Firstly, we examine relationship between Bitcoin and various currencies through all periods, thereby examining the diversifier and hedging capabilities of Bitcoin over the full sample period for each currency. We use an OLS with heteroskedasticity and autocorrelation consistent (HAC) standard errors to estimate:

$$
\Delta B_{t}=\alpha+\beta \Delta S_{t}+\gamma \Delta B_{t-1}+\varepsilon_{t}
$$

Where $\Delta B_{t}$ is the Bitcoin returns at time $t$ and $\Delta S_{t}$ is the currency spot returns at time $t$. This model enables us to further examine the hedging capabilities of Bitcoin over the full sample period. To augment the model specified in equation (12) and to address the question whether Bitcoin is a safe haven, we employ the non-temporal threshold testing procedure developed by Hansen (2000). This test sorts the currency returns in a non-temporal fashion from positive to negative returns and then detects a single unknown breakpoint. This allows us, in a non-temporal modelling framework, to endogenously identify the currency market thresholds around which Bitcoin behaviour changes and thereby testing whether Bitcoin acts as a safe haven during extreme movements in currency returns. Therefore we test the following model:

$$
\begin{array}{cc}
\Delta B_{t}=\alpha_{L}+\beta_{L} \Delta S_{t}+\gamma_{L} \Delta B_{t-1}+\varepsilon_{L, t} & \text { if } S_{t} \leq q \\
\Delta B_{t}=\alpha_{H}+\beta_{H} \Delta S_{t}+\gamma_{H} \Delta B_{t-1}+\varepsilon_{H, t} & \text { if } S_{t}>q
\end{array}
$$

Where $q$ is the currency threshold value to be estimated by the maxim and the likelihood ratio statistics over all permissible values and subscripts $L$ and $H$ denote low and high currency returns regimes. The threshold value is estimated by minimizing the sum of squared residuals generated for all tentative thresholds. Also, to ensure potential serial correlations in the errors, we include 
lags of the dependant variable as additional explanatory variables. To make sure that the residuals of the final regression do not exhibit serial correlations, we first estimate the threshold parameter and the coefficient estimates to obtain the associated residuals. We then re-order the residuals temporally and perform the test for serial correlations using the re-ordered residuals. As it turns out, including only the first lag of the dependent variable is sufficient to ensure that the errors are free of serial correlation. The heteroscedasticity character of the residuals is controlled by using the White (1980) robust standard errors.

However it could be the case that there are multiple non-temporal thresholds in the foreign currency returns and therefore extend the Hansen (2000) procedure by applying the multiple structural change analysis proposed by Bai and Perron $(1998,2003)$ to the sorted returns. This procedure searches for any number of thresholds in the returns over all possible combinations of break points. The extended model is therefore:

$$
\begin{gathered}
\Delta B_{t}=\alpha_{1}+\beta_{1} \Delta S_{t}+\gamma_{1} \Delta B_{t-1}+\varepsilon_{1, t} \quad \text { if } S_{t} \leq q_{1} \\
\vdots \\
\Delta B_{t}=\alpha_{j}+\beta_{j} \Delta S_{t}+\gamma_{j} \Delta B_{t-1}+\varepsilon_{j, t} \quad \text { if } q_{j-1}<S_{t} \leq q_{j} \\
\vdots \\
\Delta B_{t}=\alpha_{m+1}+\beta_{m+1} \Delta S_{t}+\gamma_{m+1} \Delta B_{t-1}+\varepsilon_{m+1, t} \quad \text { if } q_{m}<S_{t}
\end{gathered}
$$

Where $m$ is the number of thresholds present and $\beta_{m+1}$ measures the elasticity of Bitcoin price relative to the currency rate during the extreme market turmoil regime for the currency. Given that the number of thresholds is not known a prior, we select the optimal number of thresholds by minimizing the Bayesian Information Criterion (BIC) since Gonzalo and Pitarakis (2002) as well as Hamaker (2009) both find that the BIC shows the best performance of selecting the number of thresholds among a number of model selection criteria. Our procedure starts with the null hypothesis of 0 threshold against the alternative of 1 threshold, if it rejects, we proceed to 2 thresholds and so on. We test up to 3 thresholds and following Fatum and Yamamoto (2016), we exclude the first and the last $1 \%$ of the ordered sample for the possible threshold values.

\section{Empirical Results}

\subsection{Dynamic Conditional Correlation}

The model building strategy is to estimate several versions of the DCC and ADCC models where each specification includes a constant in the mean equation. We then include an $\operatorname{AR}(1)$ term as 
well as various GARCH models such as the GARCH(1,1) model, the GJRGARCH(1,1) model of Glosten et al (1993) to account for the asymmetry in returns as well as the EGARCH(1,1) model of Nelson (1991) which has additional leverage terms to capture asymmetry in volatility clustering. Table 3 reports the performance criteria according to the Akaike Information Criteria (AIC), the Bayesian Information Criterion (BIC) and the Hannan-Quinn Criterion (HQC) where the superior DCC or ADCC model is the AR(1) EGARCH(1,1) model since it has the smallest information criterion according to all three statistics. In this study, we only report the results for the $\operatorname{AR}(1)$ EGARCH(1,1) ADCC model since it is the superior dynamic conditional correlation model. ${ }^{6}$ Table 4 reports the AR(1) EGARCH(1,1) ADCC model regression results and we do not elaborate on the results in too much detail since the general purpose of ADCC modelling is not to derive estimates of the equations but to use these as a generating point for the conditional correlations over time. However we do note that the $\mathrm{AR}(1)$ term in the mean equation is significant, the GARCH parameters (including the asymmetry term) are generally significant and that the equations are well specified in terms of residual ARCH and other specification errors. ${ }^{7}$

The ADCCs are presented in Figure 3 where we can see quite a large variation in the correlation between AUD and Bitcoin over time however the vast majority of the correlations are greater than zero indicating that as AUD weakens in value, Bitcoin increases in value indicating the hedging benefits of Bitcoin. The CAD and CHF results are quite similar to the AUD results in that there are many periods with positive correlation indicating a weakening of the currency is positively correlate with a strengthening of Bitcoin, denoting the hedging capabilities of Bitcoin. The EUR and GBP results again show that the correlation between the currency and Bitcoin is positive indicating the potential hedging benefits of Bitcoin. Finally, the JPY figure shows very low correlation between JPY and Bitcoin, indicating that there is not much of a relationship. There are periods of positive correlation which indicate the potential hedging benefits of Bitcoin. In an attempt to summarize the ADCC results, we report in Table 5 the average ADCC over time for each currency and show that highest average ADCC is for AUD with a value of 0.0403, while the smallest is for JPY (0.0333). AUD also has the highest percentage of periods with a positive correlation $(83.49 \%)$ while the lowest number of periods with a positive correlation is EUR (72.71\%). All periods with negative correlations indicate periods where Bitcoin is a diversifier for the currencies. Therefore our results so far have showed that Bitcoin does offer intraday hedging benefits to investors at specific periods of time.

\footnotetext{
${ }^{6}$ The other models are also estimated and generate qualitatively similar results, which are available upon request from the corresponding author.

${ }^{7}$ The residuals statistics are available upon request from the corresponding author.
} 


\subsection{OLS regression}

Table 6 reports the results of the OLS with heteroskedasticity and autocorrelation consistent (HAC) standard errors analysis of the properties of Bitcoin where we report a negative relationship between Bitcoin and AUD and JPY which indicates that as Bitcoin increases in the price, the AUD and JPY strengthens in value since the currencies are per unit of US dollar. Therefore this indicates that Bitcoin is a diversifier for the AUD and JPY. We also find CHF, EUR and GBP all have positive relationships with Bitcoin indicating that when the currency value increases per US dollar (currency decreases in strength), the price of Bitcoin increases indicating the hedging properties of Bitcoin. We also show that the CAD has a zero relationship with Bitcoin indicating that Bitcoin can be a hedge for the CAD as well. Therefore these results provide further evidence of the potential benefits of Bitcoin to currency investors.

\subsection{Multiple non-temporal thresholds regression}

In order to examine whether the relationship between Bitcoin and currency changes when the depreciation of currency against the USD beyond a certain level, Table 7 reports the multiple nontemporal thresholds regression results of Equation (14). Panel A of Table 7 shows the results of testing the null hypothesis of no break against the alternatives of up to 3 breaks. As Panel A shows, there are 2 thresholds ( 3 regimes) for the EUR, the CHF, and the JPY. While there are 3 thresholds (4 regimes) for the CAD, the AUD, and the GBP. These findings confirm the existence of nonlinearity relationships between Bitcoin and the currencies, which were potentially missed by the OLS regression results in Table 6. Panel B of Table 7 shows the coefficients under different levels of market turmoil for the currencies which suggests that Bitcoin is a safe haven for the CAD, CHF and GBP currencies. In each case, there is a positive relationship between each currency and Bitcoin in periods of high or extreme market turmoil which indicates when currencies are in turmoil, Bitcoin price increase. Although Bitcoin is only a significant safe haven for the CHF. We also find that Bitcoin does not offer safe haven status during periods of extreme market turmoil for the AUD, EUR and JPY currencies. This means that in periods of high negative returns, Bitcoin does not offer safe haven status to investors. Therefore our results indicating that Bitcoin is a safe haven for the CAD, CHF and GBP currencies, but does not offer any safe haven status for the AUD, EUR and JPY.

\section{Conclusions}


In this paper we investigate the intraday hedge and safe haven interaction between Bitcoin and world currencies. The objective of our paper is two-fold. The first objective is to assess whether Bitcoin is a hedge at the intraday level. Given the rise of high-frequency trading and the high intraday volatility of Bitcoin returns, there is a need to study not only the daily hedge and safe haven properties of Bitcoin (as in Bouri et al 2017a; Bouri et al 2017b) but also whether it can at the intraday level. We employ hourly returns of Bitcoin and six developed currencies and find that the DCC results indicate that Bitcoin can been deemed a hedge at various periods of time. That is, the correlation between Bitcoin and currencies is negative and positive indicating that Bitcoin may act as a diversifier and hedge for investors. We also examine the hedge and diversifier benefits of Bitcoin over the full sample period and that the CHF, EUR and GBP are hedges while all other currencies are diversifiers.

The second objective of this paper is to assess the safe haven properties of Bitcoin and we employ the non-temporal threshold procedure of Hansen (2000) which enables us to identify periods of extreme currency returns endogenously. Our results for the non-temporal threshold analysis reveal that Bitcoin is a safe haven during high and extreme periods of market turmoil for the CAD, CHF and GBP, but fails to act as a safe haven for the other currencies. This suggests some currencies that are hedges over the full sample period, are not safe havens during extreme periods of market turmoil.

Overall, the results of our study offer three important insights. First, our results show that at the intraday level Bitcoin can be considered at hedge and diversifier for currency investors. Second, our results add to our understanding of the behaviour of Bitcoin in showing that it is a safe haven during high market turmoil in the CAD, CHF and GBP currencies, while Bitcoin offers no safe haven benefits for the other currencies. Third and more generally, our results suggest at Bitcoin does have a relationship with other financial assets and therefore future research should dig deeper and add to the understanding and modelling of the interaction between Bitcoin and other financial assets. 


\section{References}

Bai, J., Perron, P. (1998). Estimating and testing linear models with multiple structural changes. Econometrica, 66, 47-78.

Bai, J., Perron, P. (2003). Computation and analysis of multiple structural change models. Journal of Applied Econometrics, 18, 1-22.

Bariviera, A. F. (2017). The inefficiency of Bitcoin revisited: A dynamic approach. Economics Letters, $161,1-4$.

Baur, D. G., Hong, K., Lee, A. D. (2018). Bitcoin: Medium of Exchange or Speculative assets? Journal of International Financial Markets, Institutions and Money, forthcoming.

Baur, D. G., Lucey, B. M. (2010a). Is Gold a Hedge or a Safe Haven? An Analysis of Stocks, Bonds and Gold. The Financial Review, 45, 217-229.

Baur, D. G., McDermott, T. K. (2010). Is gold a safe haven? International evidence. Journal of Banking and Finance, 34, 1886-1898.

Bouri, E., Gupta, R., Tiwari, A. K., Roubaud, D. (2017a). Does Bitcoin hedge global uncertainty? Evidence from wavelet-based quantile-in-quantile regressions. Finance Research Letters, 23, 87-95.

Bouri, E., Molnár, P., Azzi, G., Roubaud, D., Hagfors, L. I. (2017b). On the hedge and safe haven properties of Bitcoin: Is it really more than a diversifier? Finance Research Letters, 20, 192198.

Brandvold, M., Molnár, P., Vagstad, K., Valstad, O. C. A. (2015). Price discovery on Bitcoin exchanges. Journal of International Financial Markets, Institutions and Money, 36, 18-35.

Bredin, D., Conlon, T., Poti, V. (2015). Does gold glitter in the long-run? Gold as a hedge and safe haven across time and investment horizon. International Review of Financial Analysis, 41, 320-328.

Cappiello, L., Engle, R. F., Sheppard, K. (2006). Asymmetric Dynamics in the Correlations of Global equity and Bond Returns. Journal of Financial Econometrics, 4, 537-572.

Cheah, E.-T., Fry, J. (2015). Speculative bubbles in Bitcoin markets? An empirical investigation into the fundamental value of Bitcoin. Economics Letters, 130, 32-36.

Ciner, C., Gurdgiev, C., Lucey, B. M. (2013). Hedges and safe havens: An examination of stocks, bonds, gold, oil and exchange rates. International Review of Financial Analysis, 29, 202-211.

Corbet, S., Lucey, B., Yarovaya, L. (2018a). Datestamping the Bitcoin and Ethereum bubbles. Finance Research Letters, 26, 81-88.

Corbet, S., Lucey, B., Urquhart, A., Yarovaya, L. (2018b). Cryptocurrencies as a financial asset: A systematic analysis. International Review of Financial Analysis, forthcoming.

Corbet, S., Meegan, A., Larkin, C., Lucey, B., Yarovaya, L. (2018c). Exploring the Dynamic Relationships between Cryptocurrencies and Other Financial Assets. Economics Letters, 165, 28-34.

Dwyer, G. P. (2015). The economics of bitcoin and similar private digital currencies. Journal of Financial Stability, 17, 81-91.

Dyhrberg, A. H. (2016). Hedging capabilities of bitcoin. Is it the virtual gold? Financial Research Letters, 16, 139-144.

Engle, R. (2002). Dynamic Conditional Correlation. Journal of Business and Economic Statistics, 20, 339-350.

Fatum, R., Yamamoto, Y. (2016). Intra-safe haven currency behavior during the global financial crisis. Journal of International Money and Finance, 66, 49-64.

Gandal, N., Hamrick J. T., Moore, T., Oberman, T. (2018). Price Manipulation in the Bitcoin Ecosystem. Journal of Monetary Economics, forthcoming.

Glosten, L. R., Jagannathan, R., Runkle, D. E. (1993). On The Relation between The Expected Value and The Volatility of Nominal Excess Return on stocks. Journal of Finance, 48, 17791801. 
Gonzalo, J. and Pitarakis, J.-Y. (2002). Estimation and model selection based inference in single and multiple threshold models, Journal of Econometrics, 110(2), pp. 319-352.

Hamaker, E. L. (2009). Using information criteria to determine the number of regimes in threshold autoregressive models, Journal of Mathematical Psychology, 53(6), pp. 518-529.

Hansen, B. E. (2000). Sample splitting and threshold estimation. Econometrica, 68, 575-603.

Kajtazi, A., Moro, A. (2019). The role of bitcoin in well diversified portfolios: A comparative global study. International Review of Financial Analysis, forthcoming.

Katsiampa, P. (2017). Volatility estimation for Bitcoin: A comparison of GARCH models. Economics Letters, 158, 3-6.

Kaul, A., Sapp, S. (2006). Y2K fears and safe haven trading of the U.S. dollar. Journal of International Money and Finance, 35, 760-779.

Lucey, B. M., Li, S. (2014). What precious metals act as safe havens, and when? Some US evidence. Applied Economics Letters, 22, 35-45.

Lucey, B. M., Vigne, S. A., et al. (2018). Future directions in international financial integration research-a crowdsourced perspective. International Review of Financial Analysis, 55, 35-49.

Manahov, V., Hudson, R., Gebka, B. (2014). Does high frequency trading affect technical analysis and market efficiency? And is so, how? Journal of International Financial Markets, Institutions and Money, 28, 131-157.

Nakamoto, S. (2008). Bitcoin: A peer-to-peer electronic cash system. https://Bitcoin.org/Bitcoin.pdf

Nadarajah, S., Chu, J. (2017), On the inefficiency of Bitcoin. Economics Letters, 150, 6-9.

Nelson, D. B. (1991). Conditional Heteroskedasticity in Asset Returns: A New Approach. Econometrica, 59, 347-370.

Newey, W. K., West, K. D. (1987). A simple, positive semi-definite, heteroskedasiticty and autocorrelation consistent covariance matrix. Econometrica, 55(3), 703-708.

O'Connor, F. A., Lucey, B. M., Batten, J. A., Baur, D. G. (2015). The financial economics of gold - A survey. International Review of Financial Analysis, 41, 186-205.

Peng, Y., Albuquerque, P. H. M., de Sá, J. M. C., Padula, A. J. A., Montenegro, M. R. (2018). The best of two worlds: Forecasting high frequency volatility for cryptocurrencies and traditional currencies with Support Vector Regression. Expert Systems with Applications, 97, 177-192.

Platanakis, E., Sutcliffe, C., Urquhart, A. (2018). Optimal vs naïve diversification in cryptocurrencies. Economics Letters, 171, 93-96.

Platanakis, E., Urquhart, A. (2019). Should Investors Include Bitcoin in Their Portfolios? A Portfolio Theory Approach. Available at SSRN: http://dx.doi.org/10.2139/ssm.3215321

Shahzad, S. J., Bouri, E., Roubaud, D., Kristoufek, L., Lucey, B. (2019). Is Bitcoin a better safehaven investment than gold and commodities? International Review of Financial Analysis, forthcoming.

Shen, D., Urquhart, A., Weng, P. (2019). Does twitter predict Bitcoin? Economics Letters, 174, 118122.

Tiwari, A. K., Jana, R. K., Das, D., Roubaud, D. (2018). Informational efficiency of Bitcoin - An extension. Economics Letters, 163, 106-109.

Urquhart, A. (2016). The inefficiency of Bitcoin. Economics Letters, 148, 80-82.

Urquhart, A. (2017). Price clustering in Bitcoin. Economics Letters, 159, 145-148.

Urquhart, A. (2018). What causes the attention of Bitcoin? Economics Letters, 166, 40-44.

White, H. (1980). A Heteroskedasticity-Consistent Covariance Matrix Estimator with a Direct Test for Heteroskedasticity. Econometrica, 84(4), 817-838. 


\section{Tables and Figures}

Table 1: Descriptive Statistics of the hourly Bitcoin returns and the foreign exchange returns. 'St.Dev' refers to the standard deviation of returns, while 'max' and 'min' denote to the maximum and minimum returns. 'Skew' refers to the skewness and 'kurt' denotes the kurtosis of returns.

\begin{tabular}{|ccccccc|}
\hline & Mean & St.Dev & Max & Min & Skew & Kurt \\
\hline Bitcoin & 0.0153 & 0.8753 & 14.3041 & -16.4390 & -1.1215 & 48.4553 \\
AUD & 0.0007 & 0.1452 & 2.5301 & -1.4487 & 0.5119 & 15.1358 \\
CAD & 0.0007 & 0.1148 & 1.8067 & -1.4247 & -0.0538 & 13.8878 \\
CHF & 0.0002 & 0.2902 & 17.4739 & -31.4227 & -55.0159 & 7882.7636 \\
EUR & 0.0004 & 0.1239 & 2.0810 & -1.7925 & 0.2271 & 23.9934 \\
GBP & 0.0010 & 0.1311 & 5.8981 & -2.2720 & 5.2512 & 238.6945 \\
JPY & 0.0000 & 0.1285 & 1.4635 & -3.0312 & -1.2451 & 32.2472 \\
\hline
\end{tabular}


Table 2: The correlation matrix between Bitcoin and the currency returns. *** indicates significance at the $1 \%$ level.

\begin{tabular}{|cccccccc|}
\hline & Bitcoin & AUD & CAD & CHF & EUR & GBP & JPY \\
\hline Bitcoin & & -0.01 & 0.00 & 0.00 & 0.00 & 0.00 & -0.01 \\
AUD & -0.01 & & $0.59^{* * *}$ & $0.12^{* * *}$ & $0.40^{* * *}$ & $0.40^{* * *}$ & $0.17^{* * *}$ \\
CAD & 0.00 & $0.59^{* * *}$ & & $0.12^{* * *}$ & $0.34^{* * *}$ & $0.36^{* * *}$ & $0.10^{* * *}$ \\
CHF & 0.00 & $0.12^{* * *}$ & $0.12^{* * *}$ & & $0.29^{* * *}$ & $0.15^{* * *}$ & $0.18^{* * *}$ \\
EUR & 0.00 & $0.40^{* * *}$ & $0.34^{* * *}$ & $0.29^{* * *}$ & & $0.49^{* * *}$ & $0.36^{* * *}$ \\
GBP & 0.00 & $0.40^{* * *}$ & $0.36^{* * *}$ & $0.15^{* * *}$ & $0.49^{* * *}$ & & $0.07^{* * *}$ \\
JPY & -0.01 & $0.17^{* * *}$ & $0.10^{* * *}$ & $0.18^{* * *}$ & $0.36^{* * *}$ & $0.07^{* * *}$ & \\
\hline
\end{tabular}


Table 3: The performance criteria of the different specifications of the DCC- and ADCC-GARCH models. The optimal model for the DCC and ADCC is in bold.

\begin{tabular}{|c|c|c|c|}
\hline Model & AIC & $\mathrm{BIC}$ & HQC \\
\hline DCC-AR(1)-GARCH(1,1) & -10.896 & -10.869 & -10.887 \\
\hline DCC-GARCH(1,1) & -10.863 & -10.839 & -10.855 \\
\hline DCC-AR(1)-GJRGARCH(1,1) & -10.895 & -10.865 & -10.886 \\
\hline DCC-GJRGARCH $(1,1)$ & -10.862 & -10.835 & -10.853 \\
\hline DCC-AR(1)-EGARCH(1,1) & -11.101 & -11.071 & -11.091 \\
\hline DCC-EGARCH $(1,1)$ & -11.070 & -11.043 & -11.061 \\
\hline ADCC-AR(1)-GARCH(1,1) & -10.939 & -10.911 & -10.930 \\
\hline ADCC-GARCH $(1,1)$ & -10.907 & -10.880 & -10.897 \\
\hline ADCC-AR(1)-GJRGARCH(1,1) & -10.939 & -10.908 & -10.929 \\
\hline ADCC-GJRGARCH $(1,1)$ & -10.905 & -10.877 & -10.896 \\
\hline ADCC-AR(1)-EGARCH(1,1) & -11.142 & -11.112 & -11.132 \\
\hline ADCC-EGARCH $(1,1)$ & -11.111 & -11.084 & -11.102 \\
\hline
\end{tabular}


Table 4: The ADCC AR(1) EGARCH(1,1) results where ' $\mu$ ' refers to the constant and ' $\omega$ ' refers to the AR(1) term in the mean equation. ' $\alpha$ ' refers to the constant in the variance equation, ' $\beta$ ' refers to the ARCH term, ' $\gamma$ ' refers to the GARCH term, ' $\lambda$ ' refers to the asymmetry term while ' $\delta$ ' refers to the shape term. ${ }^{* * *}$, $* *$ and $*$ indicate significance at the $1 \%, 5 \%$ and $10 \%$ respectively.

\begin{tabular}{|c|c|c|c|}
\hline Variable & Coefficient & Variable & Coefficient \\
\hline$\mu \mathrm{BITCOIN}$ & $0.0148^{* * *}$ & $\mu_{\mathrm{EUR}}$ & 0.0000 \\
\hline$\omega_{\text {BITCOIN }}$ & $-0.1051 * * *$ & $\omega_{\text {EUR }}$ & $-0.0541 * * *$ \\
\hline$\alpha_{\text {BITCOIN }}$ & $-0.0074 * * *$ & $\alpha_{\text {EUR }}$ & $-0.9000 * * *$ \\
\hline$\beta_{\text {BITCOIN }}$ & -0.0020 & $\beta_{\text {EUR }}$ & $-0.0358^{* * *}$ \\
\hline$\gamma_{\text {BITCOIN }}$ & $0.9928^{* * *}$ & $\gamma_{\mathrm{EUR}}$ & $0.7856^{* * *}$ \\
\hline$\lambda_{\text {BITCOIN }}$ & $0.1622^{* * *}$ & $\lambda_{\text {EUR }}$ & $0.5350^{* * *}$ \\
\hline$\delta_{\text {BITCOIN }}$ & $2.6828^{* * *}$ & $\delta_{\mathrm{EUR}}$ & $2.8386^{* * *}$ \\
\hline$\mu_{\mathrm{AUD}}$ & -0.0004 & $\mu_{\mathrm{GBP}}$ & 0.0000 \\
\hline$\omega_{\text {AUD }}$ & $-0.0384 * * *$ & $\omega_{\mathrm{GBP}}$ & $-0.0660 * * *$ \\
\hline$\alpha_{\text {AUD }}$ & $-0.0247 * * *$ & $\alpha_{\mathrm{GBP}}$ & $-0.9839 * * *$ \\
\hline$\beta_{\mathrm{AUD}}$ & 0.0008 & $\beta_{\mathrm{GBP}}$ & -0.0142 \\
\hline$\gamma_{\text {AUD }}$ & $0.9937^{* * *}$ & $\gamma_{\mathrm{GBP}}$ & $0.7606^{* * *}$ \\
\hline$\lambda_{\mathrm{AUD}}$ & $0.0732^{* * *}$ & $\lambda_{\mathrm{GBP}}$ & $0.6498^{* * *}$ \\
\hline$\delta_{\text {AUD }}$ & $3.5126^{* * *}$ & $\delta_{\mathrm{GBP}}$ & $2.6839 * * *$ \\
\hline$\mu_{\mathrm{CAD}}$ & 0.0007 & $\mu_{\mathrm{JPY}}$ & $0.0014 *$ \\
\hline$\omega_{\mathrm{CAD}}$ & $-0.0524 * * *$ & $\omega_{\mathrm{JPY}}$ & $-0.0470 * * *$ \\
\hline$\alpha_{\mathrm{CAD}}$ & $-1.1361 * * *$ & $\alpha_{J P Y}$ & $-0.0659 * * *$ \\
\hline$\beta_{\mathrm{CAD}}$ & -0.0069 & $\beta_{\mathrm{JPY}}$ & $-0.0221 * * *$ \\
\hline$\gamma_{\mathrm{CAD}}$ & $0.7397 * * *$ & $\gamma_{J P Y}$ & $0.9843^{* * *}$ \\
\hline$\lambda_{\mathrm{CAD}}$ & $0.5036^{* * *}$ & $\lambda_{\mathrm{JPY}}$ & $0.1590^{* * *}$ \\
\hline$\delta_{\mathrm{CAD}}$ & $3.1383^{* * *}$ & $\delta_{J P Y}$ & $3.0942^{* * *}$ \\
\hline$\mu_{\mathrm{CHF}}$ & 0.0007 & decal & $0.0054 * * *$ \\
\hline$\omega_{\mathrm{CHF}}$ & $-0.0806^{* * *}$ & dccb1 & $0.9910^{* * *}$ \\
\hline$\alpha_{\mathrm{CHF}}$ & $-1.0329 * * *$ & decg1 & $0.0039 * * *$ \\
\hline$\beta_{\mathrm{CHF}}$ & $-0.0320^{* *}$ & mshape & $4.0000^{* * * *}$ \\
\hline$\gamma_{\mathrm{CHF}}$ & $0.7543^{* * *}$ & & \\
\hline$\lambda_{\mathrm{CHF}}$ & $0.6201 * * *$ & & \\
\hline$\delta_{\mathrm{CHF}}$ & $2.9541 * * *$ & & \\
\hline
\end{tabular}


Table 5: The summary statistics of the ADCC estimated above, where we report the average correlation, as well as the percentage of periods with a positive and negative correlation.

\begin{tabular}{|lcccccc|}
\hline & AUD & CAD & CHF & EUR & GBP & JPY \\
\hline Ave. Corr. & 0.0403 & 0.0356 & 0.0360 & 0.0389 & 0.0346 & 0.0333 \\
\% Pos. Corr. & $83.49 \%$ & $78.28 \%$ & $72.93 \%$ & $72.71 \%$ & $74.11 \%$ & $78.50 \%$ \\
$\%$ Neg. Corr. & $16.51 \%$ & $21.72 \%$ & $27.07 \%$ & $27.29 \%$ & $25.89 \%$ & $21.50 \%$ \\
\hline
\end{tabular}


Table 6: Time-series analysis from equation (12) where $\alpha$ is the constant, $\beta$ is the currency returns and $\gamma$ is the autocorrelation term of the Bitcoin returns. $* * *, * *$ and $*$ indicates significance at the $1 \%, 5 \%$ and $10 \%$ respectively.

\begin{tabular}{|ccccccc|}
\hline & AUD & CAD & CHF & EUR & GBP & JPY \\
\hline$\alpha$ & $0.01^{* * *}$ & $0.02^{* * *}$ & $0.02^{* * *}$ & $0.02^{* * *}$ & $0.02^{* *}$ & $0.02^{* * *}$ \\
& $(2.60)$ & $(2.60)$ & $(2.60)$ & $(2.60)$ & $(2.59)$ & $(2.60)$ \\
$\beta$ & -0.04 & 0.00 & 0.01 & 0.00 & 0.03 & -0.04 \\
& $(-0.79)$ & $(0.07)$ & $(0.23)$ & $(0.03)$ & $(0.51)$ & $(-0.71)$ \\
$\gamma$ & $-0.05^{* * *}$ & $-0.05^{* * *}$ & $-0.05^{* * *}$ & $-0.05^{* * *}$ & $-0.05^{* * *}$ & $-0.05^{* * *}$ \\
No. Obs & $(-2.98)$ & $(-2.99)$ & $(-2.99)$ & $(-2.99)$ & $(-2.99)$ & $(-2.98)$ \\
Adj $R^{2}$ & 19,108 & 19,108 & 19,108 & 19,108 & 19,108 & 19,108 \\
ARCH LM & 0.00 & 0.00 & 0.00 & 0.00 & 0.00 & 0.00 \\
\end{tabular}


Table 7: Multiple non-temporal threshold analysis. $\Delta B_{t}$ is the first natural logged return for bitcoin at time $t . \alpha$ is the constant. $\Delta S_{t}$ is the first natural logged return for the currency at time $t$. The subscript $j$ represents for the states of the regimes. Threshold variable is proxied by the level of the currency against the USD. Regime 1 corresponds to portion of the sample in which the level of exchange rate from last hour is less than or equal to the threshold value. Regime 2 corresponds to portion of the sample in which the level of exchange rate from last hour is greater than the threshold value. Under the multiple threshold analysis, Regime 3 (4) refers to the depreciation of currency against the USD during the high and extreme market turmoil regime. The optimal number of thresholds is determined by BIC.

\begin{tabular}{|c|c|c|c|c|c|c|}
\hline & AUD/USD & CAD/USD & CHF/USD & EUR/USD & GBP/USD & JPY/USD \\
\hline \multicolumn{7}{|l|}{$\begin{array}{l}\text { Panel A: } \\
\text { Thresholds }\end{array}$} \\
\hline 1 & 1.2203 & 1.1968 & 1.0132 & 0.8584 & 0.6576 & 101.0700 \\
\hline 2 & 1.2222 & 1.1975 & 1.0133 & 0.8599 & 0.6577 & 101.1150 \\
\hline 3 & 1.2516 & 1.2200 & - & - & 0.6580 & - \\
\hline $\mathrm{BIC}$ & -5029.76 & -5023.08 & -5101.95 & -5126.52 & -5031.87 & -5073.12 \\
\hline Panel B: & - & - & - & - & - & - \\
\hline Coefficients & - & - & - & - & - & - \\
\hline$\alpha_{1}$ (low) & $\begin{array}{c}0.01 \\
(0.31)\end{array}$ & $\begin{array}{c}-0.02 \\
(-0.57)\end{array}$ & $\begin{array}{c}0.02^{* * *} \\
(2.97)\end{array}$ & $\begin{array}{c}0.01 \\
(0.48)\end{array}$ & $\begin{array}{c}0.01 \\
(1.11)\end{array}$ & $\begin{array}{c}-0.04 \\
(-1.17)\end{array}$ \\
\hline$\alpha_{2}$ (medium) & $\begin{array}{c}0.14 \\
(0.64)\end{array}$ & $\begin{array}{c}-.22 \\
(-0.57)\end{array}$ & $\begin{array}{l}-0.27 \\
(-0.50)\end{array}$ & $\begin{array}{c}0.19 \\
(1.47)\end{array}$ & $\begin{array}{c}-1.40^{* * *} \\
(-4.84)\end{array}$ & $\begin{array}{c}0.06 \\
(0.33)\end{array}$ \\
\hline$\alpha_{3}$ (high) & $\begin{array}{l}-0.08^{*} \\
(-1.78)\end{array}$ & $\begin{array}{l}-0.04 \\
(-1.03)\end{array}$ & $\begin{array}{c}-0.02 \\
(-0.78)\end{array}$ & $\begin{array}{l}0.02 * * \\
(2.52)\end{array}$ & $\begin{array}{c}0.01 \\
(0.13)\end{array}$ & $\begin{array}{c}0.02^{* * *} \\
(2.62)\end{array}$ \\
\hline$\alpha_{4}$ (extreme) & $\begin{array}{c}0.02^{* * *} \\
(3.29)\end{array}$ & $\begin{array}{c}0.02^{* * *} \\
(3.35)\end{array}$ & - & - & $\begin{array}{l}0.02^{* *} \\
(2.40)\end{array}$ & - \\
\hline$\Delta S_{t, 1}$ (low) & $\begin{array}{l}-0.71 * \\
(-1.86)\end{array}$ & $\begin{array}{c}0.04 \\
(0.07)\end{array}$ & $\begin{array}{c}0.00 \\
(0.02)\end{array}$ & $\begin{array}{c}0.68^{* *} \\
(2.53)\end{array}$ & $\begin{array}{c}0.00 \\
(0.01)\end{array}$ & $\begin{array}{c}-0.41 \\
(-1.54)\end{array}$ \\
\hline$\Delta S_{t, 2}$ (medium) & $\begin{array}{c}2.39 * * \\
(2.05)\end{array}$ & $\begin{array}{c}2.25 \\
(0.64)\end{array}$ & $\begin{array}{c}7.06 \\
(0.91)\end{array}$ & $\begin{array}{l}-3.59^{*} \\
(-1.84)\end{array}$ & $\begin{array}{c}16.84^{* * * *} \\
(4.97)\end{array}$ & $\begin{array}{c}-2.24 * * \\
(-2.41)\end{array}$ \\
\hline$\Delta S_{t, 3}$ (high) & $\begin{array}{c}0.11 \\
(0.28)\end{array}$ & $\begin{array}{l}-0.48 \\
(-1.33)\end{array}$ & $\begin{array}{c}0.83^{* *} \\
(2.05)\end{array}$ & $\begin{array}{l}-0.06 \\
(-0.94)\end{array}$ & $\begin{array}{c}0.27 \\
(0.31)\end{array}$ & $\begin{array}{c}-0.03 \\
(-0.53)\end{array}$ \\
\hline$\Delta S_{t, 4}$ (extreme) & $\begin{array}{l}-0.01 \\
(-0.23)\end{array}$ & $\begin{array}{c}0.01 \\
(0.20)\end{array}$ & - & - & $\begin{array}{c}0.02 \\
(0.33)\end{array}$ & - \\
\hline$\Delta B_{t-1,1}$ (low) & $\begin{array}{l}-0.08 \\
(-1.29)\end{array}$ & $\begin{array}{l}-0.06 \\
(-1.15)\end{array}$ & $\begin{array}{c}-0.05^{* * *} \\
(-2.91)\end{array}$ & $\begin{array}{c}0.01 \\
(0.16)\end{array}$ & $\begin{array}{c}-.00 \\
(-0.06)\end{array}$ & $\begin{array}{c}0.32 \\
(1.10)\end{array}$ \\
\hline $\begin{array}{c}\Delta B_{t-1,2} \\
\text { (medium) }\end{array}$ & $\begin{array}{c}0.49 * * \\
(2.37)\end{array}$ & $\begin{array}{c}0.98^{* * *} \\
(2.63)\end{array}$ & $\begin{array}{l}-1.94 \\
(-1.05)\end{array}$ & $\begin{array}{c}-0.41 * * * \\
(-3.05)\end{array}$ & $\begin{array}{c}0.76^{* * *} \\
(3.16)\end{array}$ & $\begin{array}{c}-0.81 \text { *** } \\
(-13.01)\end{array}$ \\
\hline$\Delta B_{t-1,3}$ (high) & $\begin{array}{c}0.03 \\
(0.48)\end{array}$ & $\begin{array}{l}-0.00 \\
(-0.04)\end{array}$ & $\begin{array}{c}0.02 \\
(0.24)\end{array}$ & $\begin{array}{c}-0.06 * * * \\
(-2.81)\end{array}$ & $\begin{array}{c}0.78^{* *} \\
(2.03)\end{array}$ & $\begin{array}{c}-0.04 * * \\
(-2.54)\end{array}$ \\
\hline $\begin{array}{c}\Delta B_{t-1,4} \\
\text { (extreme) }\end{array}$ & $\begin{array}{c}-0.06^{* * *} \\
(-2.99)\end{array}$ & $\begin{array}{c}-0.06 * * * \\
(-2.91)\end{array}$ & - & - & $\begin{array}{c}-0.06^{* * *} \\
(-3.20)\end{array}$ & - \\
\hline
\end{tabular}


Figure 1: The time-series graph of the foreign exchange currencies studied in this paper. Most currencies are denoted on the primary y-axis, while the JPY is the only currency attached to the secondary y-axis.

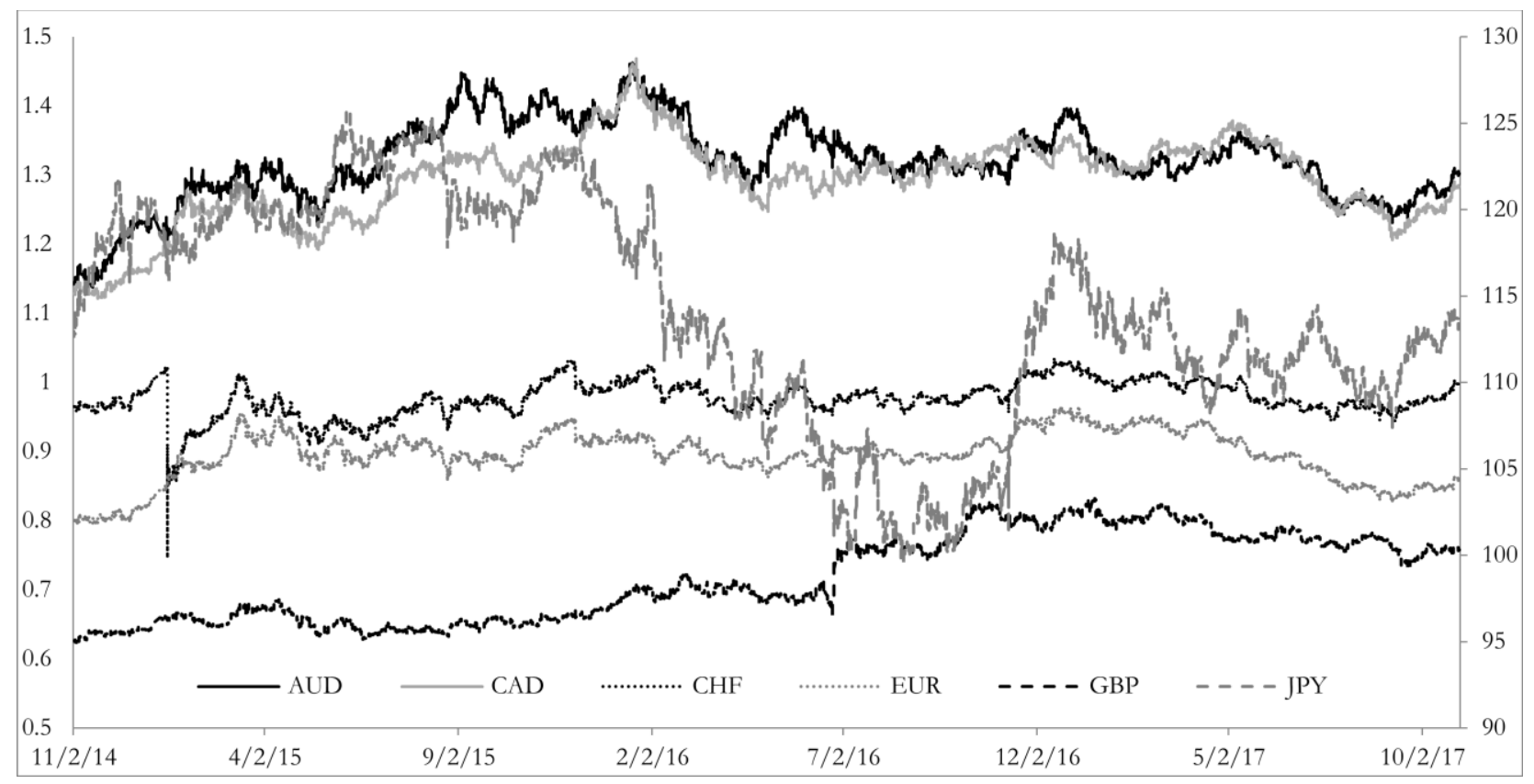

Figure 2: The time-series graph of Bitcoin.

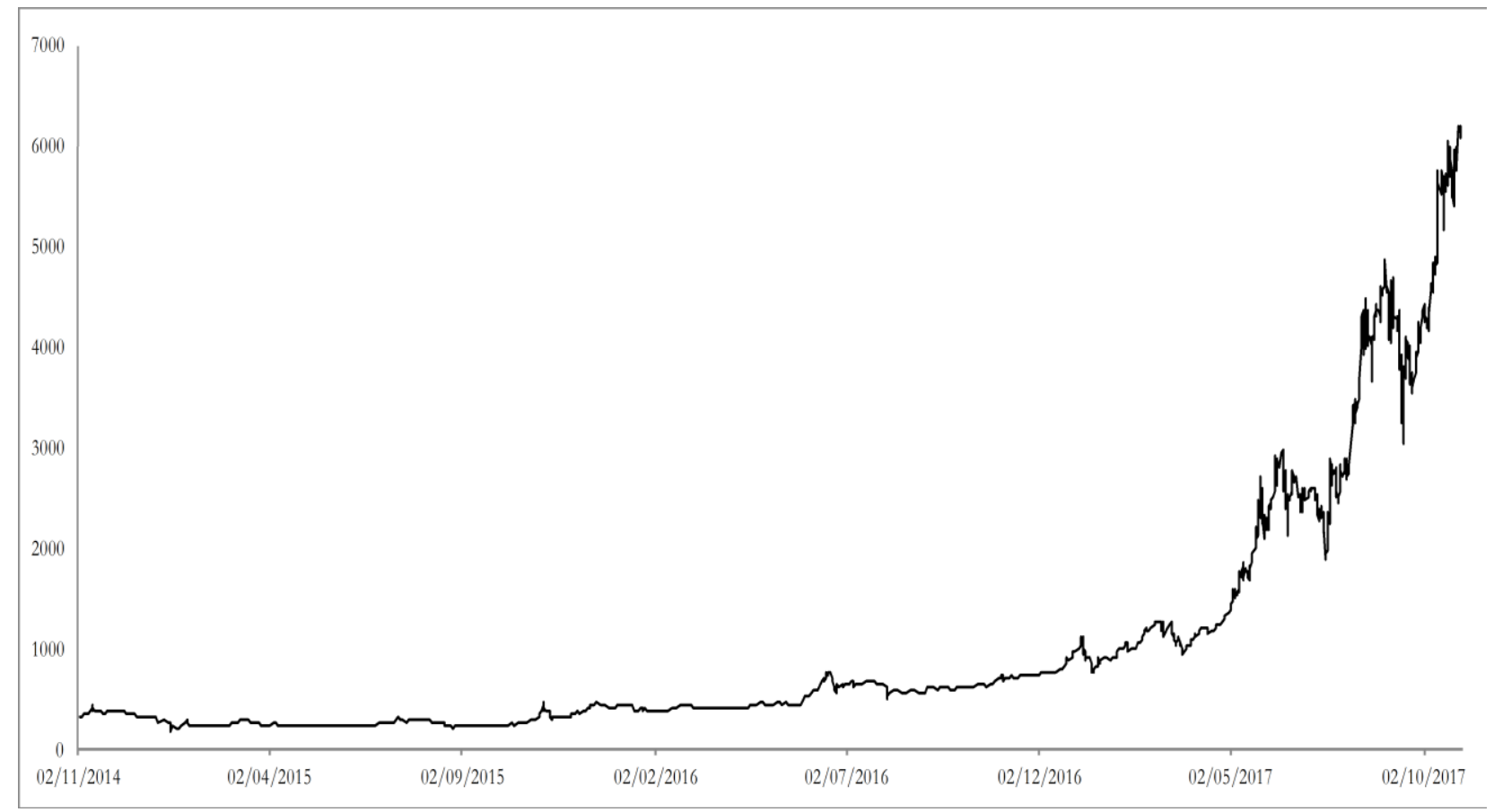


Figure 3: The optimal DCCs between the intraday currency returns and Bitcoin.
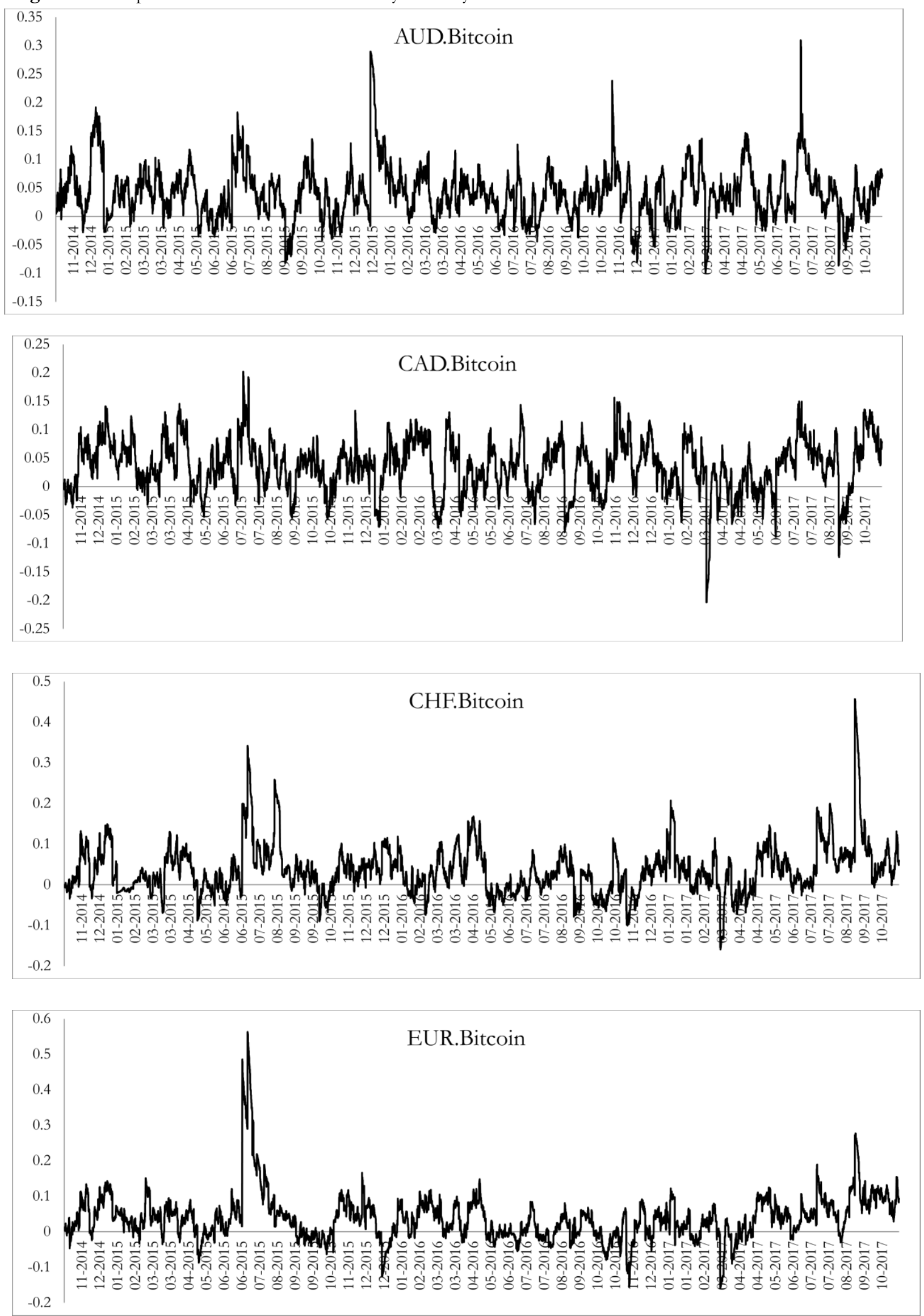

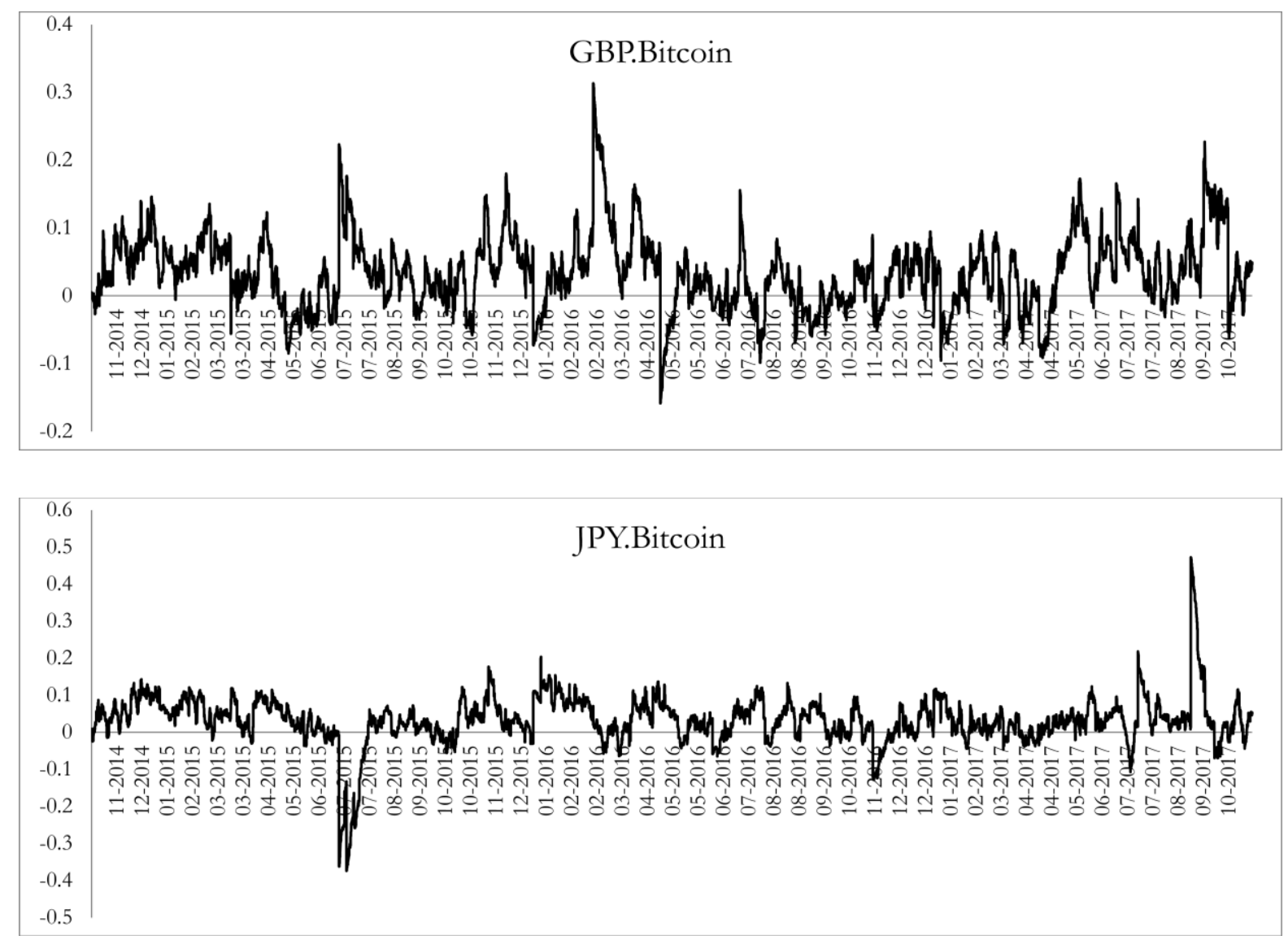